\title{
Les constatations du Comité des droits de l'homme des Nations unies en 2018 relatives à la liberté de religion en France
}

\section{Gérard Gonzalez}

\section{CpenEdition}

\section{Journals}

Édition électronique

URL : http://journals.openedition.org/rdr/1157

DOI : $10.4000 /$ rdr. 7157

ISSN : 2534-7462

Éditeur

Presses universitaires de Strasbourg

\section{Édition imprimée}

Date de publication : 9 juillet 2020

Pagination : 175-187

ISBN : 979-10-344-0065-2

ISSN : 2493-8637

\section{Référence électronique}

Gérard Gonzalez, « Les constatations du Comité des droits de l'homme des Nations unies en 2018 relatives à la liberté de religion en France », Revue du droit des religions [En ligne], 9 | 2020, mis en ligne le 18 mai 2020, consulté le 10 décembre 2020. URL : http://journals.openedition.org/rdr/1157 ; DOI : https://doi.org/10.4000/rdr.1157 


\section{LES CONSTATATIONS DU COMITÉDES DROITS DEL'HOMMEDES NATIONSUNIES EN 2018 RELATIVESÀ LA LIBERTÉ DERELIGION ENFRANCE}

\section{Gérard GONZALEZ}

Université de Montpellier, Institut de droit européen des droits de l'homme (IDEDH)

Université de Strasbourg / CNRS, Droit, Religion, Entreprise et Société (DRES)

D ar deux constatations, qui viennent grossir le passif de la politique française restreignant le port des signes religieux au regard du droit international des droits de l'homme ${ }^{1}$, le Comité des droits de l'homme des Nations unies (CDH) prend une nouvelle fois le contre-pied des juges français et européens.

\section{EMBARRASSANTES CONSTATATIONS}

Qu'il s'agisse du licenciement pour faute lourde d'une employée de crèche portant le voile ou des sanctions infligées à deux femmes portant le niqab en application de la loi du 11 octobre 2010, le CDH interpelle les politiques publiques de l'État français concernant la visibilité de l'islam tant dans l'espace

1. V. G. GonZALEz, «Bonne foi et engagements internationaux de la France en matière de liberté de religion », Revue du droit des religions, n 2, 2016, p. 171-175. 
privé $^{2}$ que public, violant ainsi la liberté de religion (1.1) des femmes musulmanes victimes à ce titre d'une double discrimination (1.2).

\subsection{UNE VIOLATION DE LA LIBERTÉ DE MANIFESTER SA RELIGION SANS BUT LÉGITIME ET DISPROPORTIONNÉE}

Dans sa constatation Baby Loup du 16 juillet 2018 (rendue publique le 10 août 2018, F. A. c. France ${ }^{3}$ ), le CDH considère que la restriction établie par le règlement intérieur de la crèche et sa mise en œuvre portent atteinte à la liberté de religion de l'auteure de la communication en violation de l'article 18 du Pacte international sur les droits civils et politiques (PIDCP) et constituent une discrimination contraire à l'article 26 du même Pacte (pts 8.9 et 8.13 infra). Mettant fin à la saga judiciaire nationale, la Cour de cassation, en assemblée plénière, avait validé le licenciement de cette employée d'une crèche refusant d'obéir à l'injonction fondée sur le nouveau règlement intérieur de l'établissement d'enlever son «voile islamique», restriction jugée suffisamment précise compte tenu de la petite taille de l'association et «justifiée par la nature des tâches accomplies par les salariés de l'association et proportionnée au but recherché » de préserver la relation des employés avec les enfants et leurs parents. Le CDH considère que le règlement intérieur permet de considérer que la restriction était bien prévue par la loi. Dans le contrôle du respect de la liberté de manifester sa religion par la requérante, c'est la seule fonction attribuée par le Comité au règlement intérieur qui a été au contraire déterminant dans le raisonnement de la Cour de cassation. Son poids est nul dans le cadre du contrôle du but légitime poursuivi et de la proportionnalité de la restriction imposée. Le licenciement pour faute lourde est dépourvu de but légitime, car «l'État partie n'a pas apporté de justification suffisante

2. La gestion privée de la crèche exclut les contraintes liées au principe constitutionnel de laiicité, mais la validation par le juge sous influence de la CJUE et la consécration par l'article L. 1321-2-1 du Code du travail issu de la loi du 8 août 2016 (loi El Khomri) autorisent l'édiction d'un règlement intérieur d'entreprise «neutralisant » à l'égard du port de signes religieux par le personnel.

3. Parmi les nombreux commentaires, pas toujours très «juridiques", de cette constatation, citons les plus pertinents fondés sur un véritable raisonnement juridique: S. HeNNETTEVAuChez, "Pour une lecture dialogique du droit international des droits humains. Remarques sur les constatations du Comité des droits de l'Homme dans l'affaire Baby Loup, et quelques réactions qu'elles ont suscitées », La Revue des droits de l'homme: http://journals. openedition.org/revdh/4643 [consulté le 15 nov. 2019] ; J. Mouly, «L'affaire Baby Loup devant le Comité des droits de l'homme: vers une révision déchirante de la jurisprudence interne?», D. 2018, p. 2097. 
qui permette de conclure que le port d'un foulard par une éducatrice de la crèche porterait atteinte aux libertés et droits fondamentaux des enfants et des parents la fréquentant» (pt 8.8). Quant à la qualification de «faute grave particulièrement stigmatisante et privative de toute indemnité de rupture ", elle est disproportionnée (pt 8.9). Le Comité rappelle au passage, point de friction essentiel avec la jurisprudence de la Cour européenne des droits de l'homme, que «le port d'un foulard ne saurait en soi être considéré comme constitutif d'un acte de prosélytisme ${ }^{4} »$.

Dans les deux affaires introduites par deux femmes verbalisées en application de la loi du 11 octobre 2010 interdisant la dissimulation du visage dans l'espace public, le Comité rend deux constatations ${ }^{5}$ identiques de violation des articles 18 (liberté de religion) et 26 (principe de non-discrimination) du Pacte. À la différence de la constatation Baby Loup, la collision avec la Cour européenne est ici frontale. Reprenant à peu de choses près les mêmes arguments que celle-ci, il en tire un constat radicalement opposé. Dans son arrêt de Grande Chambre S.A.S. c. France ${ }^{6}$, la Cour européenne avait admis le but légitime de «sûreté publique» ou de «sécurité publique» (§ 115), mais avait considéré qu' « une interdiction absolue de porter dans l'espace public une tenue destinée à dissimuler son visage ne peut passer pour proportionnée qu'en présence d'un contexte révélant une menace générale contre la sécurité publique» (§ 139). Sur ce point, les deux organes de garantie des droits humains sont en parfaite harmonie. Même constat sur «la flexibilité de la notion de "vivre ensemble" et le risque d'excès qui en découle» (\$ 122) pour la Cour européenne à laquelle répond en écho le $\mathrm{CDH}$ pour lequel «le concept du "vivre ensemble" est très vague et abstrait» (pt 8.10). Mais là où la Cour européenne abdique devant l'«ample marge d'appréciation de l'État» sur cette question de société (§ 157) et juge que la différence de traitement est justifiée (§ 161), le CDH lui ne sait pas quel droit fondamental ou liberté concrète d'autrui seraient «affectés par

4. Pt 8.9. La Cour européenne évoque «l'impact qu'un signe extérieur fort tel que le port du foulard peut avoir sur la liberté de conscience et de religion d'enfants en bas âge» (déc. 15 févr. 2001, Dahlab c. Suisse; Gde ch., 10 nov. 2005, Leyla Sahin c. Turquie, § 111 ; par opposition à un crucifix: Gde ch., 18 mars 2011, Lautsi c. Italie, § 73; 4 déc. 2008, Dogru c. France et Kervanci c. France, \& 64). Un «signe extérieur fort» peut, en soi, être perçu comme prosélyte, c'est du moins ce qui ressort implicitement de la jurisprudence de la Cour européenne.

5. CDH, constatations 17 juill. 2018 (rendues publiques le 22 oct. 2018), Sonia Yaker c. France et Miriana Hebbadj c. France.

6. CEDH, $1^{\text {er }}$ juill. 2014; sur ces questions V. le dossier publié dans cette Revue, $\mathrm{n}^{\circ} 2,2016$, notamment P. Rolland, "L'arrêt S.A.S. c. France de la Cour européenne des droits de l'homme», p. 47-60. 
le fait que certaines personnes évoluent dans l'espace public avec le visage couvert », ni «pourquoi ces droits seraient affectés "de manière déloyale" par le port du voile intégral et non par la dissimulation du visage dans l'espace public par de nombreux autres moyens non visés par la loi ». Surtout, il observe justement que «le droit d'interagir avec n'importe quel individu dans l'espace public et le droit de ne pas être troublé par le port du voile intégral par d'autres personnes ne sont pas protégés par le Pacte» (pt 8.10). Cette constatation est assortie de deux opinions dissidentes qui insistent sur le but légitime de sécurité publique dans un contexte d'attentats terroristes perpétrés par des islamistes intégristes, frange à laquelle semblent d'office rattachées les femmes ayant une pratique aussi radicale. Yadh Ben Achour, auteur de l'une des deux opinions dissidentes, insiste aussi sur la validité du but légitime du «vivre ensemble» et sur le caractère proportionné de l'interdiction assortie d'amendes légères.

Les deux constats sont particulièrement sévères qui trouvent l'État défaillant à justifier cumulativement (là où la Cour européenne s'en tient généralement au constat du premier critère d'acceptabilité de l'ingérence manquant) du but légitime et de la proportionnalité de la mesure contestée.

\subsection{DES INGÉRENCES CONSTITUTIVES DE DISCRIMINATIONS INTERSECTIONNELLES}

Dans les deux cas (affaire Baby Loup et interdiction du niqab), le Comité retient une discrimination intersectionnelle (Baby Loup) ou croisée (niqab) à l'égard des auteures des communications. Là encore l'effet cumulatif de ces constats accroît le ressenti liberticide des mesures contestées.

Dans l'affaire Baby Loup, le règlement intérieur, de peu de poids dans le contrôle du respect de la liberté de religion de l'auteure, réapparaît plus évidemment dans le contrôle du respect du principe de non-discrimination. Malgré son caractère neutre avancé par le gouvernement, le Comité considère qu'il est à l'origine d'un "traitement différencié», car il "affecte de façon disproportionnée les femmes musulmanes, telles que l'auteure, faisant le choix de porter un foulard» (pt 8.12), soit une discrimination indirecte qui pour être acceptable doit poursuivre un but légitime et être proportionnée. Compte tenu de ses conclusions sur la liberté de religion, on comprend aisément que ce ne sera pas le cas et le Comité peut conclure que «le licenciement de l'auteure basé sur le règlement intérieur de la crèche qui prévoit une obligation de neutralité du personnel dans l'exercice de ses tâches, et sur le Code du travail, ne reposait pas sur un critère raisonnable 
et objectif et constitue donc une discrimination intersectionnelle basée sur le genre et la religion» (pt 8.13). Ce constat d'une double discrimination ${ }^{7}$ interpelle directement la Cour européenne qui, à propos du port du foulard, s'interroge «sur l'effet prosélytique qu'il peut avoir dès lors qu'il semblait être imposé aux femmes par un précepte religieux difficilement conciliable avec le principe d'égalité des sexes ${ }^{8} »$. Avec cette constatation, c'est toute la doctrine des établissements areligieux souhaitant fonder leur organisation sur le principe de neutralité qui est remis en question du point de vue de la conception universelle des droits de l'homme qui heurte de plein fouet la conception régionale européenne en voie de développement. Quant au caractère discriminatoire de l'interdit posé par la loi de 2010, le principe de neutralité de la loi fait long feu, le Comité observant que «bien que rédigée en termes généraux, la loi $\mathrm{n}^{\circ}$ 2010-1192 prévoit des exceptions pour la plupart des contextes de dissimulation du visage dans l'espace public, limitant ainsi l'applicabilité de l'interdiction au voile islamique intégral et guère plus» (pt 8.13). Il souligne le caractère potentiellement contre-productif de la loi qui pourrait aboutir à confiner ces femmes chez elles. L'interdiction assortie de sanctions pénales «affecte de façon disproportionnée l'auteure en tant que femme musulmane qui choisit de porter le voile intégral, et introduit entre elle et les autres personnes qui couvrent parfois leur visage dans l'espace public de façon légale une distinction qui n'est ni nécessaire ni proportionnée à un intérêt légitime et qui est donc déraisonnable» et constitue « une forme de discrimination croisée basée sur le sexe et la religion, en violation de l'article 26 du Pacte» (pt 8.17). Finalement, le CDH aboutit à une conclusion qui aurait été celle de la Cour européenne si, respectueuse du principe de subsidiarité, celle-ci ne s'était pas retranchée derrière la marge d'appréciation de l'État pour lui concéder in extremis un constat de non-violation.

7. Sur la notion de discrimination intersectionnelle, V. S. HennetTe-VAuchez, art. cit.; V. aussi J. Mouly, art. cit.

8. Dahlab précit., repris dans Leyla Sahin précit., § 64, Dogru et Kervanci précit., § 64. Il est vrai que ces affaires concernent exclusivement le milieu scolaire et n'ont pas pour cadre une entreprise ou association privée. La Cour européenne pourrait être plus conciliante. Par ex., CEDH, 18 sept. 2018, Lachiri c. Belgique à propos de l'expulsion d'une salle d'audience d'une partie civile voilée. Mais le paragraphe 46 de l'arrêt interroge, la Cour affirmant qu'«il ne résulte pas des pièces de la procédure que l'objectif poursuivi en l'espèce par l'exclusion de la requérante de la salle d'audience aurait été la préservation de la neutralité de l'espace public», ce dont on peut déduire a contrario qu'il pourrait en aller autrement si cet objectif était clairement établi. 
Le Comité a pris ici ses responsabilités. Après la constatation Baby Loup, qui s'aventurerait à parier que la jurisprudence de la Cour de Luxembourg ${ }^{9}$, relayée par la Cour de cassation ${ }^{10}$, promouvant la neutralité au sein de l'entreprise via son règlement intérieur, atténuée dans sa rigueur par l'obligation de l'employeur de proposer à son salarié un poste moins «visible» plutôt que de le licencier, se hisse au niveau de compatibilité requis par le Comité avec l'exigence d'un but légitime à cette restriction et avec le principe de proportionnalité?

Dans les «affaires du niqab», le Comité est allé plus loin que dans sa constatation de 2012 relative à l'application de la loi du 15 mars 2004 encadrant, en application du principe de laïcité, le port de signes ou de tenues manifestant une appartenance religieuse dans les écoles, collèges et lycées publics. Par cette constatation, il considérait que «le principe de laicité est en soi un moyen par lequel un État partie peut s'efforcer de protéger la liberté religieuse de l'ensemble de la population et que la loi $n^{\circ}$ 2004-228 a été adoptée en réaction à des affaires réelles dans lesquelles il y a eu ingérence dans la liberté religieuse des élèves et même, dans certains cas, des menaces contre leur sécurité physique [et que la loi] sert les objectifs de protection des droits et libertés d'autrui, de l'ordre public et de la sécurité publique», mais sanctionnait son application disproportionnée conduisant au renvoi définitif d'un élève de l'enseignement public. Validée dans son principe, la loi était remise en cause seulement dans son application la plus stricte ${ }^{11}$. Ici, c'est la loi de 2010, dépourvue de tout but légitime selon le Comité, qui est directement mise en cause. On connaît les interrogations et divergences qu'a engendrées l'arrêt S.A.S. de la Cour européenne ${ }^{12}$. D'un point de vue strictement juridique, les droits humains ont horreur du vide résultant d'une interdiction générale et absolue couvrant tout l'espace

9. CJUE, Gde ch., 14 mars 2017, $\mathrm{n}^{\circ}$ C -188/15, Asma Bougnaoui, Association de défense des droits de l'homme (ADDH) c. Micropole SA; ${ }^{\circ}$ C-157/15, Samira Achbita, Centrum voor gelijkheid van kansen en voor recismebestrijding c. G4S Secure Solutions NV; V. F. LARONZE et M. Schмitт, «La religion et le travail au milieu du gué européen. Sur la méthode juridico-politique des avocats généraux près la CJUE», Revue du droit des religions, $\mathrm{n}^{\circ} 3$, 2017, p. 125-137.

10. Cass. soc., 22 nov. 2017, $\mathrm{n}^{\circ}$ 13-19855: Revue du droit des religions, $\mathrm{n}^{\circ}$ 6, 2018, p. 153 159, obs. G. GonZALEZ.

11. Au risque néanmoins de poser un problème d'application tenant de la quadrature du cercle. CDH, constatation $1^{\text {er }}$ nov. 2012 (rendue publique le 4 févr. 2013), Bikramjit Singh $c$. France.

12. Pour une illustration topique, V. les commentaires croisés de G. GonZalez et G. HaARSCHER, "Consécration jésuitique d'une exigence fondamentale de la civilité démocratique? Le voile intégral sous le regard des juges de la Cour européenne», RTDH 2015, p. 220-233. 
public $^{13}$ et il faut de très fortes raisons, impérieuses et incontestables, pour en valider l'édiction. Mais aujourd'hui, la remise en question des valeurs communes de l'idéal républicain, de la laïcité, de l'égalité des sexes oblige à dépasser cette dimension strictement juridique et à promouvoir, plus qu'hier, l'image d'une démocratie fondée sur ces valeurs, apte à se défendre, quitte à saper quelques-uns de ses fondements juridiques les plus classiques et à passer pour promouvoir un universalisme des droits humains ethnocentré. La Cour européenne a choisi cette voie d'une façon qui peut paraître laborieuse sur le strict plan du discours de la méthode juridique. Le Comité tient ferme la voie du classicisme d'une conception individualiste des droits humains pourtant éloignée de la conception théocratique de ceux qui s'en réclament. Une nouvelle quadrature du cercle en quelque sorte dont l'universalisme des droits humains ne sort pas indemne, mais dont il faut bien tenir compte.

\section{LA VALEUR DISCUTÉE DES CONSTATATIONS DU CDH}

Confusion avec le Conseil des droits de l'homme des Nations unies, rabaissement du CDH au rang d'organe purement consultatif distillant des avis aussi peu utiles qu'inconséquents, rien n'a été épargné à cet organe onusien qui a eu l'aplomb de «condamner» la France, pays autoproclamé des droits de l'homme ${ }^{14}$. La posture française à l'égard du Comité, au regard notamment de l'application de la loi de 2004, a déjà été critiquée dans cette revue ${ }^{15}$. Depuis, deux constatations plus tard, le même réquisitoire peut être dressé. La posture française (2.2) ignore très largement la portée de son engagement dans le Pacte sur les droits civils et politiques et son Protocole facultatif (2.1).

\subsection{QUELLE PORTÉE POUR L'ENGAGEMENT DE LA FRANCE DANS LE PACTE ET SON PROTOCOLE FACULTATIF RELATIF AUX COMMUNICATIONS ÉMANANT DE PARTICULIERS ${ }^{16}$ ?}

La France a ratifié le Pacte international sur les droits civils et politiques le 4 novembre 1980 et son Protocole facultatif, le 17 février 1984, reconnaissant

13. CEDH, 23 févr. 2010, Ahmed Arslan c. Turquie, § 49.

14. Pour les détails de cette cabale médiatique à laquelle se sont jointes des voix d'universitaires, V. S. Hennette-Vauchez, art. cit.

15. V. note 1.

16. Sur cette question, on consultera l'article de S. HenNETTE-VAuChez précit.; aussi T. Onillon, «La valeur des constatations du Comité des droits de l'homme de l'ONU», AJDA 2019, p. 1040 et s. 
la compétence du Comité pour examiner des communications émanant de particuliers se prétendant victimes d'une violation d'un des droits énoncés par le Pacte et qui prévoit que «le Comité fait part de ses constatations à l'État partie intéressé et au particulier» (art. 5 \& 4). Elle s'est ainsi soumise dans l'application de ces traités aux principes universellement reconnus ${ }^{17}$ du libre consentement, de la bonne foi et à la règle pacta sunt servanda. La question est de savoir ce que signifie exactement, en termes d'engagements, la notion de «constatation» qui n'est évidemment ni une décision ni un arrêt, le Comité n'étant pas un organe juridictionnel. Datant de 2008, l'observation générale $(\mathrm{OG}) \mathrm{n}^{\mathrm{o}} 33$ intitulée «Les obligations des États parties en vertu du Protocole facultatif se rapportant au Pacte international sur les droits civils et politiques» clarifie, du point de vue du Comité qui en est l'auteur, cette question. Elle souligne que, bien que le $\mathrm{CDH}$ ne soit pas un organe judiciaire, «les constatations qu'il adopte en vertu du Protocole facultatif présentent certaines caractéristiques principales d'une décision judiciaire», en particulier un processus «marqué notamment par l'impartialité et l'indépendance des membres du Comité, l'interprétation réfléchie du libellé du Pacte et le caractère déterminant de ses décisions» (pt 11). Selon l'observation générale, quand le Comité conclut à une violation, il «énonce une réparation» (pt 12). Ainsi dans l'affaire Baby Loup, le CDH indique que «l'État parti [sic] est tenu, entre autres: d'indemniser l'auteure de manière adéquate et de prendre des mesures de satisfaction appropriées, incluant une compensation pour la perte d'emploi sans indemnités et le remboursement de tout coût légal, ainsi que de toute perte non pécuniaire encourue par l'auteure en raison des faits de l'espèce $[\ldots]$ de prendre toutes les mesures nécessaires pour prévenir des violations similaires à l'avenir» (pt 10); il souhaite aussi «recevoir de l'État partie, dans un délai de 180 jours, des renseignements sur les mesures prises pour donner effet aux présentes constatations» (pt 11). Dans l'affaire du niqab, il décide que l'État «est tenu, notamment, d'accorder à l'auteure des mesures de satisfaction appropriées, y compris une indemnisation financière correspondant au préjudice subi [...] de veiller à ce que des violations analogues ne se reproduisent pas, y compris en réexaminant la loi no 2010-1192 à la lumière des obligations découlant du Pacte, en particulier des articles 18 et $26 »$ (pt 10). Il faut noter cependant que l'énoncé de la réparation n'est aucunement prévu par le Pacte ni par le Protocole, mais le $\mathrm{CDH}$ s'appuie sur la mention à l'article $2 \S 3 \mathrm{a}$ du Pacte de l'obligation de

17. Codifiés par la convention de Vienne sur le droit des traités du 23 mai 1969 que la France n'a pas ratifiée, ces principes s'imposent en tant que coutume internationale même aux États n'ayant pas adhéré à ladite convention. 
l'État de «garantir que toute personne dont les droits et libertés reconnus dans le Pacte auront été violés disposera d'un recours utile» (pt 14), utilité qui ne saurait être avérée sans l'indication de mesures de réparations qui, en droit international, ont pour fonction d'effacer toute trace de l'acte illicite constitutif d'une violation d'une règle du droit international, ici un traité. L'observation générale rappelle enfin le principe général selon lequel «le devoir de coopérer avec le Comité découle de l'application du principe de la bonne foi à l'observation de toutes les obligations conventionnelles» (pt 15). Force est donc de constater que, malgré les efforts du Comité, l'État n'est pas dénué d'arguments pour résister à l'application de ses constatations et des mesures de réparation qu'elles indiquent, la sanction de la bonne foi, dans un système de garantie des droits de l'homme qui ne se fonde pas sur le principe de réciprocité, demeurant toute symbolique. Conscient de cette situation, le $\mathrm{CDH}$ termine son observation générale $\mathrm{n}^{\circ} 33$ en soulignant que lorsque «l'État partie a rejeté les constatations du Comité, en totalité ou en partie, après avoir participé à la procédure et alors que ses arguments avaient été pleinement examinés [...] le Comité considère toujours que le dialogue avec l'État partie se poursuit en vue de la mise en œuvre de la décision». Le Rapporteur spécial chargé du suivi des constatations entretient ce dialogue et rend compte régulièrement au Comité de l'évolution de la situation.

On peut objecter que le Comité, auteur de l'OG no 33 et donc juge et partie, outrepasse les compétences qui lui sont expressément reconnues par le Pacte et le Protocole. Il manque à ces textes, pour établir a minima l'autorité de la chose interprétée aux constatations du $\mathrm{CDH}$, l'équivalent de l'article 32 de la Convention européenne des droits de l'homme selon lequel «la compétence de la Cour s'étend à toutes les questions concernant l'interprétation et l'application de la Convention et de ses protocoles qui lui seront soumises $[\ldots]$ ». Pour le $\mathrm{CDH}$, la procédure des rapports présentés par les États, dépourvue de tout effet juridique, est essentiellement dialogique (art. 40 du Pacte); quant aux constatations issues de plaintes individuelles, le Comité «fait part» à l'État et au particulier (art. $5 \S 4$ du Protocole), ce qui devrait contribuer à «mieux assurer l'accomplissement des fins du Pacte international relatif aux droits civils et politiques et l'application de ses dispositions » selon le préambule du Protocole. On peut donc soutenir que les constatations du Comité ressortissent à la soft law dont les effets juridiques demeurent limités hors les cas d'une accumulation participant au processus de formation de la coutume internationale. Il reste cependant que les observations et constatations du Comité ne sont pas dépourvues d'effets dans l'établissement d'un standard minimum de garantie 
des droits humains. La Cour européenne des droits de l'homme sait s'en servir comme levier pour faire évoluer son interprétation de la Convention ${ }^{18}$, mais il est aussi vrai qu'elle sait s'en affranchir volontairement, affirmant sans ambages que «lorsque des dispositions de la Convention et du PIDCP sont presque identiques, les interprétations que le $\mathrm{CDH}$ et la Cour font d'un même droit fondamental peuvent ne pas toujours correspondre ${ }^{19}$ ». Néanmoins, la France, en ratifiant le Protocole facultatif, s'est engagée à ne pas faire la sourde oreille aux interprétations du Comité qui lui déplaisent. Il est paradoxal (ou hypocrite, c'est selon...) de se placer volontairement sous la «surveillance» d'un organe dont la compétence est facultative, avec d'ailleurs une faculté de «dénonciation » organisée par l'article 12 du Protocole, et d'en refuser tous les effets qui contreviennent à la vision nationale ou européenne promue par ailleurs. L'acceptabilité d'une ingérence dans le cadre d'un système de protection des droits humains relève d'un standard minimum propre à ce système qui peut, même si c'est regrettable, ne pas correspondre au standard élaboré par un autre système de garantie. On pourrait penser que les systèmes régionaux, notamment européens compte tenu de la maturité « démocratique» des États les composant, sont en mesure d'établir des standards plus élevés de protection que les systèmes universels de protection. Pourtant cette logique est renversée: le système universel de protection établit un standard beaucoup plus élevé que le système européen de plus en plus bridé par le principe de subsidiarité $e^{20}$ qui pourrait bien rendre durable la prééminence du système universel de garantie sur le système régional européen.

\subsection{LA POSTURE FRANÇAISE}

Les autorités politiques et, pour partie, juridictionnelles françaises, restent sourdes à ces constats, avec même parfois une pointe de mépris. Rien à voir avec la dévotion, certes récente, à l'égard de la Cour européenne telle

18. Par ex. CEDH, Gde ch., 4 févr. 2005, Mamatkulov et Askarov c. Turquie (mesures provisoires); 7 juill. 2011, Bayatyan c. Arménie (liberté de religion et service militaire obligatoire). V. F. Tulkens, S. VAn Drooghenbroecck et F. KRenc, «Le soft law et la Cour européenne des droits de l'homme: questions de légitimité et de méthode », RTDH 2012, p. 433 et $s$.

19. CEDH, Gde ch., 4 avr. 2018, Correia de Matos c. Portugal, § 135 (à propos du droit de se défendre soi-même, la Cour prend le contrepied de la constatation CDH du 28 mars 2006, Correia de Matos c. Portugal - les faits de l'arrêt CEDH remontant à 2010 attestent que le Portugal n'est pas non plus un champion au regard du respect des constatations du Comité).

20. Illustré éloquemment par l'arrêt de Grande Chambre Correia de Matos précit. 
qu'exprimée dans le discours présidentiel de Strasbourg d'octobre 2017. Il est vrai que la représentation au sein du Comité composé d'experts indépendants est essentiellement assurée par des universitaires alors que, pour la France au moins, juges judiciaires (Yves Bot comme avocat général à la CJUE, André Potocki actuel juge français à la Cour EDH) et administratifs surtout (Jean-Claude Bonichot, juge à la CJUE, Jean-Paul Costa, ancien président de la Cour EDH, Stéphane Gervasoni, juge au Tribunal UE, Ronny Abraham, ancien juge à la CIJ...) se partagent généralement les fonctions de juge dans les diverses juridictions régionales et universelles. Ceci explique peut-être en partie cela! La défiance vient peut-être aussi du fait que, à la différence des juridictions régionales ou européennes, lorsque le Comité examine une communication individuelle, le règlement intérieur prévoit que le membre du Comité élu au titre d'un État partie «ne prend [pas] part à l'examen d'une communication par le Comité a) si la communication concerne l'État partie pour lequel il ou elle a été élu $[\ldots]^{21} »$. On peut en tirer deux conséquences diamétralement opposées: soit c'est un gage supplémentaire d'indépendance de l'organe en question; soit c'est une entorse dommageable au principe selon lequel le juge «national» est, par sa présence, en mesure d'éclairer ses collègues sur telle ou telle spécificité du droit national. En tout cas, c'est un prétexte supplémentaire de défiance à l'égard d'une décision qui n'a pu bénéficier d'un tel éclairage s'agissant notamment de la complexité des rapports de l'État français à la religion via le principe de laïcité, dont, même lorsqu'il n'est pas directement impliqué, la grande ombre plane sur toutes ces affaires. Ainsi, dans une question parlementaire écrite, M. Evrard s'interroge sur un événement qui irait «à l'encontre de ce que la communauté des Nations est en droit d'attendre de l'ONU. [Dans] l'affaire de la crèche Baby Loup, le comité des droits de l'Homme de l'ONU émet un avis sur les suites judiciaires de l'affaire comme étant une atteinte aux libertés, c'est-à-dire une condamnation de la laïcité française. Est-ce dans ses compétences?»; ce à quoi le ministre répond que

«le gouvernement français considère que les constatations du Comité des droits de l'Homme et des autres comités en matière de protection des droits de l'Homme ne sont pas contraignantes. [...] [il] considère notamment que le terme "constatation", traduit en anglais par "views" et en espagnol par "observaciones", décrivant les décisions du Comité, tel qu'il est employé à l'article 5 \& 4 du Protocole facultatif instaurant les communications individuelles, signifie, sans la moindre ambiguité, qu'il s'agit d'une recommandation faite à un État par le Comité chargé 
d'interpréter le Pacte et non d'une décision impérative qu'il y aurait lieu de mettre à exécution ${ }^{22} »$.

Le Conseil d'État est dans la même ligne, soit qu'il écarte l'invocation des dispositions du Pacte au motif que les «droits à pension [...] ne relèvent pas de la catégorie des droits protégés par le Pacte ${ }^{23}$ », le Comité ayant pourtant constaté une violation de celui-ci ${ }^{24}$, soit qu'il déclare que «les constatations émises par le comité des droits de l'homme institué par l'article 28 du pacte international relatif aux droits civils et politiques ne revêtent pas de caractère contraignant à l'égard de l'État auquel elles sont adressées et ne sauraient donc lier les juridictions françaises ${ }^{25} »$. La Cour de cassation, dans sa formation Commission de révision, confirme que «les constatations du Comité des droits de l'homme ne revêtent pas de caractère contraignant à l'égard de l'État auquel elles sont adressées» et "ne constituent pas en elles-mêmes des faits nouveaux » au sens de l'article 622 du Code de procédure pénale ${ }^{26}$. Pourtant, c'est de la Cour de cassation que pourrait venir un assouplissement de la posture française plus en accord avec les engagements internationaux et le principe de bonne foi. Lors de l'audience solennelle d'installation du 3 septembre 2018, le Président Louvel a jeté un pavé dans la mare en affirmant que «dans l'ordre international, ce rôle de gardien des libertés est aussi partagé avec d'autres juridictions ou organismes issus des traités auxquels notre pays a choisi d'adhérer, juridictions et organismes qui peuvent entrer en désaccord avec notre Cour de cassation et lui imposer leur propre vision de la défense des libertés ». Après l'évocation attendue des cours européennes, il citait le CDH et la récente constatation Baby Loup, soulignant que «même si cette constatation n'a pas, en droit, de force contraignante, l'autorité qui s'y

22. Rép. min. $\mathrm{n}^{\circ}$ 12215: JOAN, 5 févr. 2019, p. 1139 (l'intitulé de la question est «actions partisanes de l'ONU»!)

23. CE, ass., avis, 15 avr. 1996, M Me Doukouré: RFDA 1996, p. 1239 note DhommEaux et RFDA 1997, p. 966 note F. SUDRE.

24. CDH, constatation 3 avr. 1989, comm. no 196/1985, Ibrahim Gaye c. France.

25. CE, 11 oct. 2001, no 238849; 3 nov. 2003, no 239559; 5 mai 2006, no 242713. D'autres juridictions suprêmes intervenant dans le domaine administratif sont plus audacieuses. Ainsi, dans une affaire de violences domestiques impliquant des négligences des autorités espagnoles, le Tribunal suprême espagnol impose-t-il l'application des constatations du Comité pour l'élimination des discriminations à l'égard des femmes et le dédommagement, jusqu'ici refusé, des victimes: Tribunal suprême, 17 juill. 2018, nº 1263/2018: V. C. Gutiérez EsPADA, «La aplicación en España de los dictámenes de comités internacionales: la STS 1263/2018, un importante punto de inflexión », Cuadernos de derecho transnacional, vol. 10, $\mathrm{n}^{\circ} 2$, oct. 2018, p. 836-851 : https://doi.org/10.20318/cdt.2018.4406 [consulté le 15 nov. 2019].

26. Cass., 10 déc. 2015, nº 14REV017: Bull. nº 5. 
attache de fait constitue un facteur nouveau de déstabilisation de la jurisprudence qui vient perturber, aux yeux des juges du fond, le rôle unificateur de notre Cour, qui plus est au niveau le plus élevé de son assemblée plénière» et qu'il «nous faut réfléchir en permanence à de nouveaux mécanismes nécessaires pour intégrer les directives croissantes d'origines diverses, et pas toujours cohérentes, que reçoit notre Cour, et qui, encore une fois, exercent nécessairement une incidence forte sur la motivation de ses arrêts ${ }^{27} »$. Faute de mieux, cette voie moyenne convient. Elle se situe dans la logique des États qui, bien qu'ayant ratifié le Pacte et son Protocole facultatif, rejette les constatations du Comité. Comme le préconise l'OG no 33, le dialogue doit être maintenu avec ces États ou, à tout le moins, avec leur pouvoir judiciaire qui peut ne pas avoir les mêmes états d'âme que l'exécutif et le législatif. Sous l'autorité du Président Louvel, cette option s'avère envisageable, mais on en ignore pour l'instant les conséquences. S'agissant de l'application de la loi de 2004 dans les écoles, collèges et lycées publics, le juge judiciaire n'est pas compétent sauf situation exceptionnelle d'application par un établissement d'enseignement privé de la loi de 2004 dans son règlement intérieur ${ }^{28}$. En revanche, le juge judiciaire a prise directe sur l'application d'un règlement intérieur d'entreprise et de la loi de 2010. Dans le premier cas, un dialogue est en effet possible sur la portée du règlement intérieur et la sanction qui y est associée; dans le second, compte tenu de la condamnation de la loi de 2010 en elle-même par le Comité, on peine à imaginer la nature du dialogue à engager, la seule solution, inenvisageable en l'état actuel des choses, consistant à écarter l'application de cette loi pour satisfaire à l'engagement universel français. On le voit, le compromis n'est pas simple, dans un cas au moins il semble introuvable, d'autant que, dans l'affaire du niqab, l'État peut s'appuyer sur la jurisprudence de la Cour européenne. Une chose est sûre en tout cas: plus que jamais l'universalisme des droits humains s'enracine de plus en plus profondément dans la pure utopie.

27. Peu après ces déclarations cependant, la Cour de cassation a évité de donner corps à cet engagement lorsqu'elle a été saisie de la demande du Comité des droits des personnes handicapées de surseoir à l'arrêt de l'alimentation et de l'hydratation entérales de Vincent Lambert pendant son examen de la communication dont il a été saisi. Pour ce faire, elle a considéré que les juridictions judiciaires n'étaient pas compétentes en l'absence de voie de fait, le droit à la vie n'étant pas une liberté au sens de l'article 66 de la Constitution (Cass., ass., 28 juin 2019).

28. Cass. $1^{\text {re }}$ civ., 21 juin 2005, nº 02-19.831, Benmehania: AJDA 2005, p. 1863. 